\title{
Enhanced MIMO Influence on LTE-Advanced Network Performances
}

\author{
Suad Kasapovic ${ }^{1}$, Samira Mujkic ${ }^{1}$, Samra Mujacic $^{1}$ \\ ${ }^{I}$ Department of Telecommunications, Faculty of Electrical Engineering, University of Tuzla, \\ Franjevacka br. 2, 75000 Tuzla, Bosnia and Herzegovina \\ suad.kasapovic@untz.ba
}

\begin{abstract}
Introduction of LTE-Advanced network brings a series of new techniques as well as an improvement of the existing ones, which contributes to better performance in relation to LTE network. Enhanced MIMO represents one of LTE-Advanced network's most significant techniques which enable considerably better performance. Spectral efficiency and throughput are important system performance indicators. The paper aims to estimate LTE-Advanced network performance by applying an enhanced MIMO. The results verify consistency with given the analytical values, as well as which system parameter has the greatest influence on the achieved results. When enhanced MIMO is applied, several performance testing scenarios have been created depending on the parameter which was analysed for the given scenario, namely: LTE-Advanced channel scenario, antenna configuration, number of HARQ retransmissions and the type of subframe mode.
\end{abstract}

Index Terms-Enhanced MIMO, LTE-Advanced, Spectral efficiency, Throughput fraction.

\section{INTRODUCTION}

The first release of LTE provides peak rates of $300 \mathrm{Mb} / \mathrm{s}$, a radio-network delay of less than $5 \mathrm{~ms}$, a significant increase in spectrum efficiency compared to previous cellular systems, and a new flat radio-network architecture designed to simplify operation and reduce cost [1]. The minimum requirements for peak spectral efficiencies are $15 \mathrm{bit} / \mathrm{s} / \mathrm{Hz}$ for downlink and $6.75 \mathrm{bit} / \mathrm{s} / \mathrm{Hz}$ for uplink [2]. However, LTE network does not meet some specific International Mobile Telecommunications-Advanced (IMTAdvanced) demands, which doesn't make it part of fourth generation networks. LTE-Advanced is a 3GPP candidate for IMT-Advanced radio access which needs to meet demands regarding $1 \mathrm{~Gb} / \mathrm{s}$ peak data rate and $30 \mathrm{bit} / \mathrm{s} / \mathrm{Hz}$ spectral efficiency in the downlink while demands for the uplink are $500 \mathrm{Mb} / \mathrm{s}$ peak data rate and $15 \mathrm{bit} / \mathrm{s} / \mathrm{Hz}$ [3]. These highest peak data rates so far are not possible to achieve with $20 \mathrm{MHz}$ bandwidth and $4 \times 4$ MIMO scheme that LTE network supports. Due to this fact, new techniques are introduced in LTE-Advanced network, the most important of which are Carrier aggregation and Enhanced MIMO. Spectrum allocation, which can be achieved in LTE-Advanced network with carrier aggregation, is essential for achieving peak data rate of transmission and is guaranteed by LTE-Advanced network while $30 \mathrm{bit} / \mathrm{s} / \mathrm{Hz}$ and $15 \mathrm{bit} / \mathrm{s} / \mathrm{Hz}$ spectral efficiency in downlink and uplink respectively is gained with $20 \mathrm{MHz}$ bandwidth and $8 \times 8$ MIMO scheme in downlink and $4 \times 4$ in uplink [4].

The paper presents evaluation of enhanced MIMO influence (antenna configuration, HARQ, environment and duplex scheme) on LTE-Advanced network in downlink. To ascertain conditions under which the best performance is achieved, when enhanced MIMO is applied, parameters values have been changed and based on that, different scenarios of the same model will be formed and system performance will be compared. Performance evaluation of LTE and LTE-Advanced network is shown in [5] where the influence of bandwidth, CQI values and antenna configuration on throughput and spectral efficiency are analysed. Analysis of throughput fraction in the function of SNR for LTE-Advanced network is presented in the paper [6], but using $2 \times 2$ MIMO scheme with different bandwidth and modulation technique in uplink and downlink. Furthermore, influence of different parameters on LTEAdvanced network throughput is analysed in [7] using carrier aggregation, which ensures bandwidth extension above $20 \mathrm{MHz}$, but maximum to $100 \mathrm{MHz}$. Parameter analysis in [4] was conducted by taking into account the influence of modulation, bandwidth and code rate on throughput. In this paper, the focus is on the analysis of LTE-Advanced network performance by applying an enhanced MIMO with an $8 \times 8$ MIMO model. Taking into consideration the number of assessed parameters and by default the number of the test scenarios, it can be concluded that the paper offers a comprehensive analysis of the LTEAdvanced network performance unlike [4]-[6] where the analyses were made using considerably fewer parameters.

The following section presents the characteristics and features of the enhanced MIMO. The third section provides an analytical calculation for peak spectral efficiency and throughput fraction. LTE-Advanced model with $8 \times 8$ MIMO scheme for conducting simulations and testing the influence of the applied enhanced MIMO is shown in the fourth part. In the fourth part additional scenario of the basic model is formed with the aim of ascertaining which system parameter values of the analysed model result in best performance during the appliance of the enhanced MIMO. The results of the analyses are shown in the fifth while discussion of the results are given in the sixth part. At the end of the paper there is a conclusion. 


\section{ENHANCED MIMO}

Advanced MIMO techniques in release 10 include higher order MIMO (up to $8 \times 8$ ) and multi-user MIMO (MUMIMO) beamforming. Increasing the antenna array size to 8 effectively doubles the peak data rate compared to releases $8 / 9$ and is the only solution to meet the ITU-R requirement of $30 \mathrm{bit} / \mathrm{s} / \mathrm{Hz}$ spectral efficiency [8]. In LTE-Advanced network it is possible to conduct transport in two transport blocks, each with special modulation and coding scheme MCS. Spatial multiplexing and transmit diversity are two basic modes of LTE network while MIMO modes which are used in LTE-Advanced network are:

- Single-User MIMO (SU-MIMO);

- Multi-User MIMO (MU-MIMO);

- Cooperative MIMO - eNodeB cooperation from different cells so as to serve one user.

The basic operating principle of eNodeB during the appliance of different MIMO modes of LTE-Advanced network is shown in the Fig. 1.

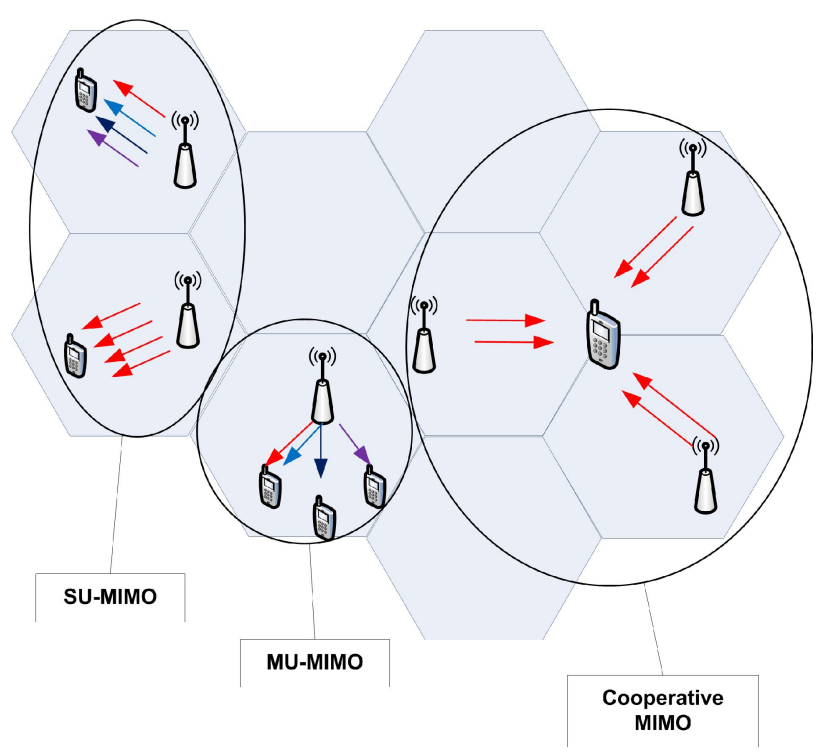

Fig. 1. MIMO modes in LTE-Advanced network.

For realization of the mentioned MIMO modes in LTEAdvanced network, the new transmission mode transmission mode 9 (TM9) is formed, which supports Single User (SU) MIMO and Multi User (MU) MIMO and enables dynamic switching between these two modes. Dynamic switching between SU-MIMO and MU-MIMO mode is introduced in Rel-10. The change of mode is done on a subframe depending on channel condition and traffic [9]. In LTE-Advanced network with the enhanced MIMO, the maximum number of supported layers for spatial multiplexing is extended from 4 layers, which was possible in LTE network to 8 layers in downlink. In order to support 8 layer transmission, LTE-Advanced extends the concept of downlink Demodulation Reference Symbol-DM-RS to 8 layers [10]. MU-MIMO allocates multiple users in one timefrequency resource to exploit multi-user diversity in the spatial domain, which results in significant gains over SUMIMO [11] and enables sharing all 8 transmit antennas among multiple users. Using Zero-forcing precoder, better removal of interference is achieved, which ensures enhancement of MU-MIMO, which is why the aforementioned precoder is used in all testing scenario simulations. Furthermore, for ascertaining the importance and difference of the MIMO modes, for different users, different MIMO modes are selected namely: spatial multiplexing and transmit diversity. LTE-Advanced network achieves enhancement in precoding based on the codebook, especially in the case involving closed loop where transmit parameters are chosen based on feedback. Given that with 8 antenna transmission the codebook has a larger content, the selection of the preferred precoding matrix index (PMI) is made more difficult due to longer estimates. LTE-Advanced MIMO scheme also uses only two code words because it has been established that the increase of code words does not enhance performance to a great degree. Figure 2 shows the mapping of code words into layers.

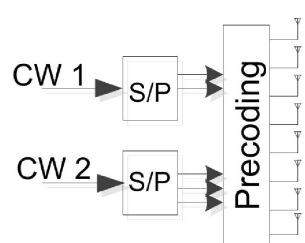

5 Layers

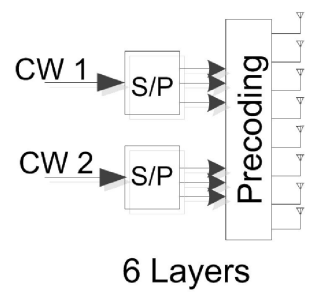

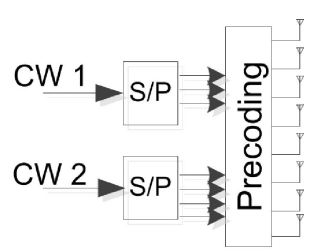

7 Layers

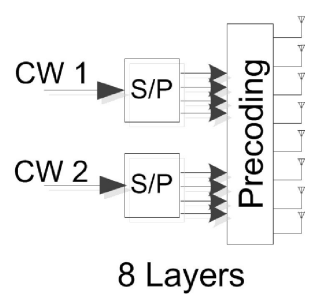

Fig. 2. Code words to layer mapping.

However, the performance achieved through MIMO depends heavily on a large number of parameters such as: receiving and transmitting antennas, reference signals, coding methods, channel estimation, and their delivery from transmitters to receivers [12].

The received vector, signal of a $i$-th mobile user in a specific interval of time in downlink of MU-MIMO system is calculated as

$$
Y_{i}=H_{i} M_{i} x_{i}+n_{i},
$$

where $H_{i}$ is flat-fading channel matrix of $i$-th user, $M_{i}$ is modulation matrix which does beamforming and $x_{i}$ is a data vector of arbitrary dimension $S_{i}$ which in turn represents a number of parallel data streams sent to $i$-th user. Noise is represented by the $n_{i}$ vector. The matrix $H_{i}$ dimensions are $M_{R, i} \times M_{T}$, where $M_{T}$ is a number of transmit antennas on the base station while $\mathrm{M}_{\mathrm{R}, \mathrm{i}}$ refers to the number of antennas of $i$-th user, with $\mathrm{S}_{\mathrm{i}}>2$.

\section{ANALYTiCAl CAlculation OF PSE AND TF}

The peak spectral efficiency (PSE) is basically the highest theoretical data rate normalized by bandwidth assignable to a single mobile station assuming error-free conditions [13], [14]. Peak spectral efficiency of LTE-Advanced system can be mathematically expressed as

$$
P S E=\frac{R}{B}
$$


where $\mathrm{R}$ is the highest theoretical data rate and $\mathrm{B}$ is the bandwidth. Given that $\mathrm{R}$ is theoretically the highest rate, the paper will analyse the achieved throughput instead of $\mathrm{R}$.

However, the paper doesn't do the direct analysis of the achieved throughput; throughput fraction is analysed instead. Throughput fraction is a relation between the achieved and peak spectral efficiency for given conditions and it is expressed in percentage. Mathematical formula for throughput fraction can be expressed as follows

$$
T F=\frac{S E}{P S E}
$$

where TF is throughput fraction, SE achieved spectral efficiency, PSE is peak spectral efficiency.

To determine peak spectral efficiency and by default throughput fraction, it is necessary to estimate throughput under the conditions in which the maximum value is achieved. We will consider $20 \mathrm{MHz}$ bandwidth at which the maximum number of resource blocks allocated to the user is 100. Each resource block consists of 12 consecutive subcarriers in the frequency domain and one $0.5 \mathrm{~ms}$ slot in the time domain and consists of $7 \times 12=84$ resource elements in the case of a normal cyclic prefix and $6 \times 12=$ 72 resource elements in the case of an extended cyclic prefix [15]. Every frame is comprised of 20 slots so that the total number of resource elements allocated to the user per frame is $\mathrm{N}_{\mathrm{RE}}=\mathrm{N}_{\mathrm{RB}} \times \mathrm{N}_{\mathrm{SC}} \times \mathrm{N}_{\mathrm{OSy}} \times \mathrm{N}_{\mathrm{S}}$ where $\mathrm{N}_{\mathrm{RE}}$ is the number of resource elements, $\mathrm{N}_{\mathrm{RB}}$ is the number of resource blocks, $\mathrm{N}_{\mathrm{SC}}$ is the number of subcarriers, $\mathrm{N}_{\mathrm{OSy}}$ is the number of OFDM symbols and $\mathrm{N}_{\mathrm{S}}$ is the number of slots per frame.

Apart from data, resource elements are used to transmit different control information necessary for the transmission of reference signals, broadcast transmission, synchronisation and control signalling [13]. So it is also important to estimate the number of resource elements per frame necessary for transmission of control information in the case of $20 \mathrm{MHz}$ bandwidth and when enhanced MIMO is applied. So for $8 \times 8$ configuration there are 32 reference signals per resource block pair in total and based on that it can be concluded that 32000 resource elements are necessary for reference signals while 240 resource elements per frame are necessary for Physical Broadcast Channel (PBC). A synchronisation channel ( $\mathrm{SCH}$ ) transmits primary and secondary synchronisation signals (PSS and SSS) and for their transmission 288 resource elements per frame are necessary. For control signalling the first OFDM signal in each sub frame of all resource blocks is used, but four resource elements of each resource block are not used as they are reserved for reference signals, so the total number of resource elements per frame necessary for control signalling is 8000 .

By adding previous estimates we get the total number of resource elements used for transmission of control information, reference signals, control of broadcast transmission and synchronisation $\mathrm{N}_{\mathrm{CRE}}=\mathrm{N}_{\mathrm{RS}}+\mathrm{N}_{\mathrm{BCH}}+$ $\mathrm{N}_{\mathrm{SS}}+\mathrm{N}_{\mathrm{CSL} 1 / 2}=40528$, where $\mathrm{N}_{\mathrm{CRE}}$ is the total number of control RE, $\mathrm{N}_{\mathrm{RS}}$ is number RE for reference signals, $\mathrm{N}_{\mathrm{BCH}}$ is number RE necessary for broadcast transmission, $\mathrm{N}_{\mathrm{SS}}$ is the number of RE necessary for the transport of primary and secondary synchronisation signals and $\mathrm{N}_{\mathrm{CSL} 1 / 2}$ represents necessary $\mathrm{RE}$ for control signalling on $1^{\text {st }}$ and $2^{\text {nd }}$ layer. Many simulations and studies show that there is $25 \%$ overhead used for controlling and signalling [14].

Given its support and the usage of 64 QAM modulation, six bits are mapped in one OFDM symbol. $8 \times 8$ antenna configuration enables transmission on 8 layers so that taking into consideration this information peak spectral efficiency is

$$
\mathrm{PSE}=\frac{\mathrm{R}}{\mathrm{B}}=\frac{\mathrm{T}}{\mathrm{B}}=\frac{\mathrm{N}_{\mathrm{L}} \times \mathrm{M}\left(\mathrm{N}_{\mathrm{RE}}-\mathrm{N}_{\mathrm{CRE}}\right)}{\mathrm{B}} \approx 30,6 \mathrm{bit} / \mathrm{s} / \mathrm{Hz},
$$

where $\mathrm{T}$ is throughput of the case model and $\mathrm{M}$ number of bits that are mapped in one OFDM symbol.

\section{Simulation OF $8 \times 8$ Mimo Model AND TeST SCENARIOS}

Figure 3 shows a block scheme of LTE-Advanced system with $8 \times 8$ MIMO scheme, FDD mode and fading environment along with a closed loop through which ACK/NACK and PMI information is transmitted. Influence of fading on the appearance of the packet error has been surveyed through packet error probability [16]. The EDA (electronic design automation) environment for electronic system level design, SystemVue, was used for presentation, analysis and conduction of simulations.

SystemVue has the ability to simulate multi-channel MIMO scenarios that may be less convenient to configure as actual hardware measurements, thus providing a simulationbased alternative for early algorithmic and functional verification. SystemVue provides LTE-Advanced MIMO channel models for prediction throughput simulation of LTE-A. SystemVue is specifically designed to mitigate the complexity of the challenge facing $8 \times 8$ MIMO device developers, yet are flexible enough to enable troubleshooting of systems and components [17]. The system shown below is a model used for conducting simulations of the formed test scenarios.

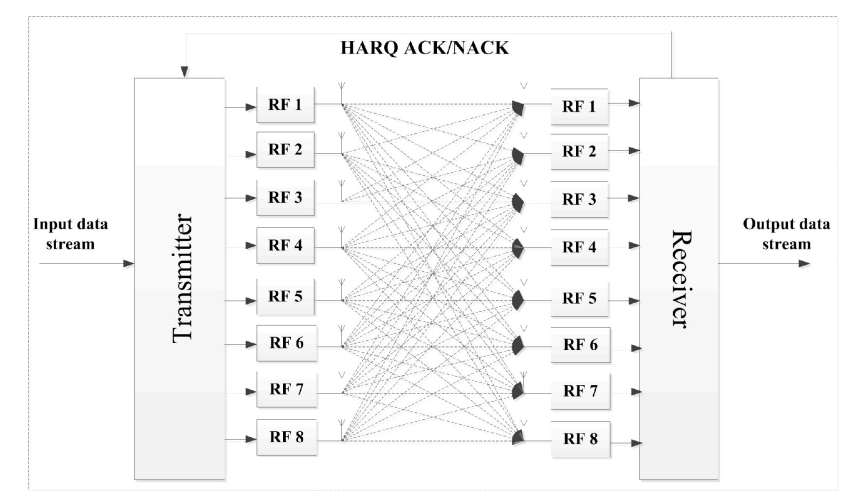

Fig. 3. LTE-Advanced system with 8x8 MIMO for downlink.

Table I shows the basic parameters of the general scenario (Scenario 1) of the LTE-Advanced system.

Relevant parameters - those that have the greatest influence on throughput and spectral efficiency are: applied antenna configuration, a number of HARQ retransmissions, duplex type and LTE-Advanced channel scenario. Scenarios shown in Tables II are formed based on modification of one 
of the named relevant parameters whilst retaining other parameters as in the general scenario. Some scenarios have sub scenarios as well due to change of the same parameter in different ways so as to analyse what parameter value influences throughput the most.

TABLE I. VALUES FOR BASIC PARAMETERS OF THE GENERAL SCENARIO

\begin{tabular}{|c|c|}
\hline Name & Value \\
\hline Duplex mode & FDD \\
\hline Bandwidth & $10 \mathrm{MHz}$ \\
\hline Modulation & 64 QAM \\
\hline Channel model - LTE-A scenario & InH \\
\hline MIMO mode & UE1: SM \\
& UE2-6: TD \\
\hline Transmission mode & UE1: TM 9 \\
& UE2-6: TM 2 \\
\hline Number of layers & UE1: 8 \\
& UE2-6: 2 \\
\hline Carrier Frequency & $2000 \mathrm{MHz}$ \\
\hline Code rate & $1 / 2$ \\
\hline Number of HARQ process & 8 \\
\hline Number of maximum HARQ retransmission & 4 \\
\hline Allocation of RB for UE1 & {$[0,21]$} \\
\hline Number of subframes for simulation & 2000 \\
\hline
\end{tabular}

All simulations are carried out for the scenario Indoor Hot which is intended for serving a small number of users in closed areas. Given that all simulations took into consideration the results UE1-UE6, the applied scenario Indoor Hot provides the best results regarding spectral efficiency and throughput fraction. Apart from eNodeB, LTE-Advanced network also uses relay nodes $(\mathrm{RN})$ in the access network so as to achieve better performance and one of the open questions is the optimal location for RNs that can provide maximum capacity [18]. The organisation of relay nodes depends on environment conditions and based on which parameter influence is necessary to eliminate or decrease there are several different applicable scenarios.

Scenario 2.1 was created to verify system performance with Umi relay nodes scenario applied (scenario for application in micro cells of urban areas) and other parameters retained from the general scenario. With Umi scenarios base station and user terminal antennas should be placed below the peaks of the surrounding buildings that is they should be 10 meters away from the roof.

In Scenario 2.2 LTE-Advanced scenario of application of relay nodes is changed to $\mathrm{RMa}$ (a scenario convenient for rural areas) while all other parameters from the general scenario are retained. For Rural macro-RMa- scenario user distribution should be $100 \%$ outdoor while base station antennas are positioned at $35 \mathrm{~m}$ above the roof, which enables propagation characteristic modelling with mobile devices movig at the speed of $120 \mathrm{~km} / \mathrm{h}$.

Each LTE-Advanced scenario has a task to model a propagation channel between base and mobile station antennas for all links, both servicing and interference ones. However, this paper does not analyse propagation characteristics.

To ascertain the influence of enhanced MIMO on the extent of network performance enhancement, apart from the analysis of $8 \times 8$ antenna scenario (Scenario 1), which is supported with introduction of the new technique, scenarios with $4 \times 4$ and $2 \times 2$ antenna configuration are analysed
(Scenario 3.1 and Scenario 3.2).

Given that all simulations conduct throughput fraction and spectral efficiency measurement in fading environment and with a closed loop sending HARQ ACK/NACK feedback is enabled. It is found that HARQ protocols employed in MAC layer of LTE systems, although consuming resources for retransmission of erroneously received data, contribute to improve both cell spectral efficiency, independent of the maximum number of retransmissions permitted [19]. To ascertain how the change of the number of HARQ retransmissions influences LTEAdvanced network performance Scenario 4 is created where the number of maximum HARQ retransmissions is decreased from 4 from the general scenario (Scenario 1) to 2.

In Scenario 5 only change of duplex scheme from FDD to TDD is done so as to compare performance achieved for different duplex schemes. Formed test scenarios are shown in Table II.

TABLE II. TEST SCENARIOS.

\begin{tabular}{|c|c|}
\hline Test scenario & Modified parameter \\
\hline $\begin{array}{c}\text { Scenario 1 } \\
\text { (General) }\end{array}$ & $\begin{array}{c}\text { Bandwidth 10 MHz, 64 QAM, max HARQ } \\
\text { retranssmision 4, LTE-A scenario InH }\end{array}$ \\
\hline Scenario 2.1 & LTE-A scenario: Umi \\
\hline Scenario 2.2 & LTE-A scenario: Ra \\
\hline Scenario 3.1 & MIMO 4 4 4 \\
\hline Scenario 3.2 & MIMO 2 2 \\
\hline Scenario 4 & Number of maximum HARQ retransmission: 2 \\
\hline Scenario 5 & Duplex scheme: TDD \\
\hline
\end{tabular}

\section{RESUlts ANALYSIS}

\section{A. Influence of different LTE-Advanced Channel Scenarios on Results}

The achieved spectral efficiency for scenarios with different LTE-Advanced channel is shown in Fig. 4.

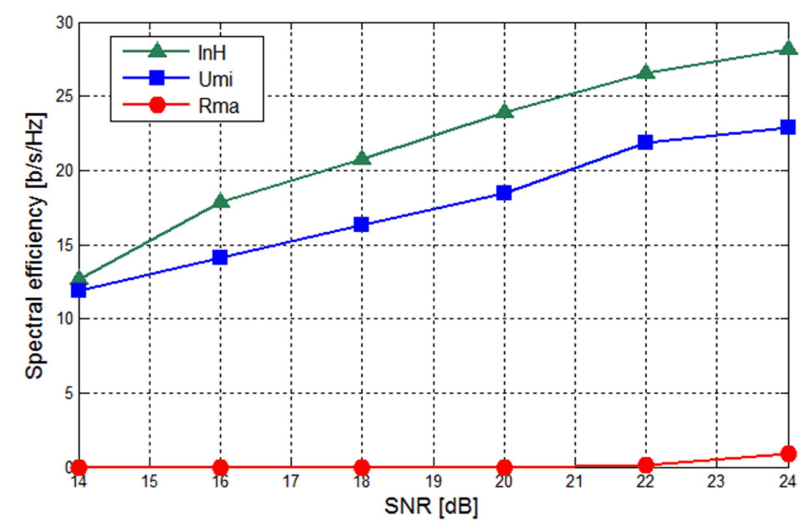

Fig. 4. Spectral efficiency in relation to SNR for scenarios with different LTE-A scenarios.

By analysing spectral efficiency and throughput fraction for different LTE-Advanced channel scenarios, that is, 3 formulated scenarios - Scenario 1, Scenario 2.1 and Scenario 2.2 hypothesis that default scenario, Indoor Hot, which was applied in all simulations, yield the best results has been proved. Slightly lower results are achieved with Umi while considerably low results are achieved with RMa relay nodes scenario for both when spectral efficiency and throughput fraction were analysed and it is below the IMTA requirements. 


\section{B. Influence of MIMO Technique Antenna Configuration on the Results}

Figure 5 and Fig. 6 provide results of spectral efficiency and throughput fraction achieved with the application of different MIMO scheme configurations. A considerably higher spectral efficiency is achieved when enhanced MIMO $8 \times 8$ antenna configuration is applied, which enables transmission on 8 layers then it is the case with $4 \times$ 4 and $2 \times 2$ antenna configurations. It was found that an increase of throughput and spectral efficiency depends on the number of antennas and is proportional to the function $\min (\mathrm{nTx} ; \mathrm{nRx})[5]$. However, by analysing the achieved results for throughput fraction gained through the application of different antenna configurations, it is evident that higher SNR gives results close to the maximum values, for the given conditions of each scenario.

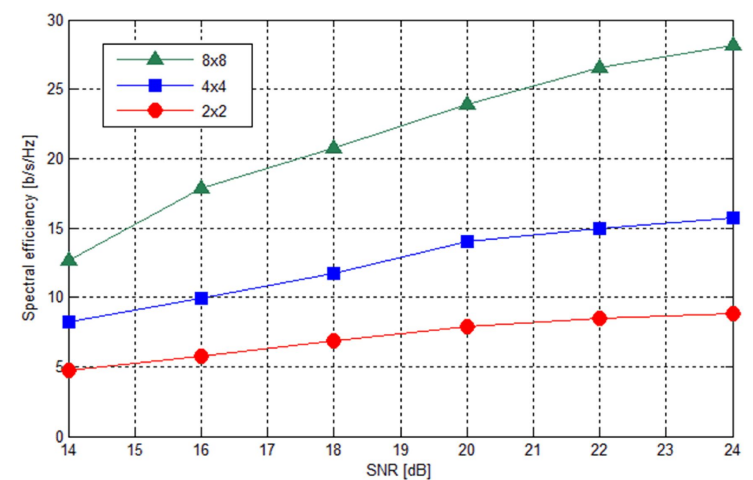

Fig. 5. Spectral efficiency in relation to SNR for scenarios with different MIMO scenarios.

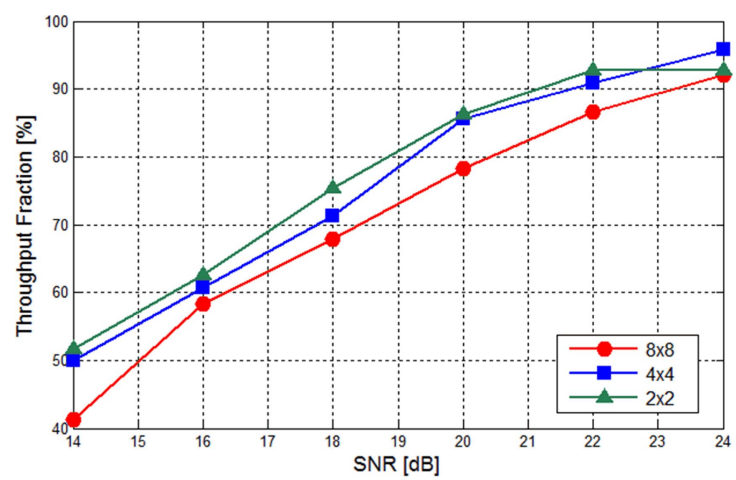

Fig. 6. Throughput fraction in relation to SNR for scenarios with different MIMO scenarios.

It is precisely the analyses of this type - when maximum values for specific scenarios are not the same and one wants to examine how those values are attained, assessment of throughput fraction enables analyses and comparisons.

Analysis of spectral efficiency for different MIMO scenarios confirmed considerably better LTE-A performance when $8 \times 8 \mathrm{MIMO}$ model is applied, while the analysis of throughput fraction reveals that with higher SNR values each scenario gets closer to the maximum values.

\section{Influence of the Change of the Number of Maximum $H A R Q$ Retransmissions on the Results}

Figure 7 shows values of spectral efficiency for Scenarios 1 and 4.

HARQ technique helps in reducing effect of multipath fading channel. Therefore, the article analysed the influence of different number of HARQ retransmissions on the LTE-
Advanced network performance. Based on the comparison of the achieved results for the considered scenarios it is evident that with the greater number of HARQ retransmissions better results are achieved, but by increasing SNR (more than $22 \mathrm{~dB}$ ) those results are achieved with the smaller number of HARQ retransmissions so there is no need to use HARQ procedure, due to lower performance impact while processing a large number of HARQ retransmissions is demanding.

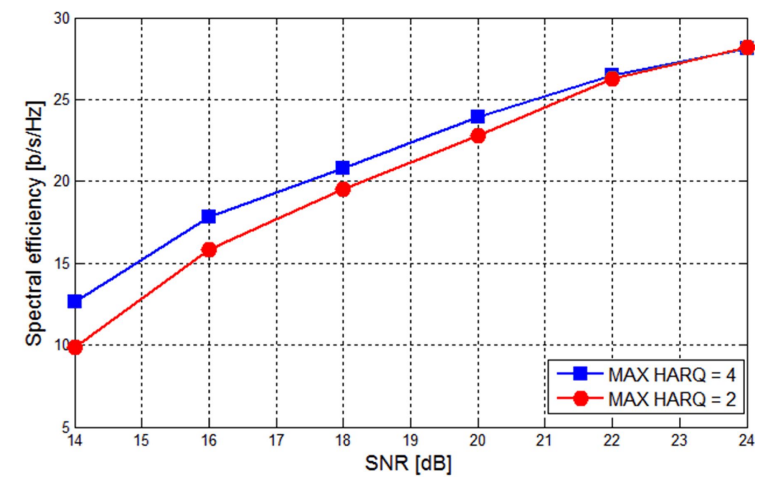

Fig. 7. Spectral efficiency in relation to SNR for scenarios with different max HARQ number.

\section{Influence of Duplex Scheme on the Results}

Frame structure with FDD and TDD duplex scheme differ considerably and due to that fact it was interesting to analyse the influence of differences on LTE-Advanced network performance. By changing duplex scheme from the general scenario from FDD in TDD, Scenario 5 was formed. Figure 8 shows the achieved spectral efficiency for scenarios 1 and 5 .

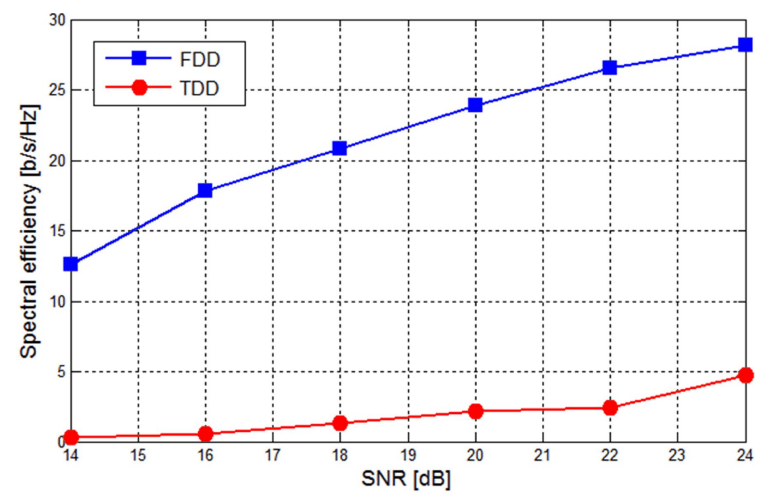

Fig. 8. Spectral efficiency in relation to SNR for scenarios with different duplex schemes.

Comparison of simulations results reveals that the results achieved through applying FDD duplex scheme are considerably higher in terms of throughput fraction and spectral efficiency. The ratio between the achieved results for these two scenarios is slightly smaller but significant nonetheless as the results of both throughput fraction and spectral efficiency are five times higher when FDD duplex scheme is applied in comparison to the results reached for TDD duplex scheme. The immense disparity in the achieved results for these two scenarios is a consequence of higher overhead in TDD frame.

\section{DISCUSSION}

The achieved results prone that the best performance is 
achieved with $20 \mathrm{MHz}$, while with higher SNR the results with $10 \mathrm{MHz}$ are almost the same. High-order modulation is more prone to errors due to its higher sensitivity to interference, noise and channel estimation errors, it is therefore useful only when SINR is sufficiently high. Simulation results have also confirmed that with lower SNR approximate values of spectral efficiency are achieved for the analysed scenarios while with the increase of SNR above $20 \mathrm{~dB}$ the scenario with 64 QAM modulation achieves considerably better results. With the new LTE-A network techniques, primarily the enhanced technique of interference coordination and interference removal technique better, SNR conditions are ensured and, therefore, support for higher modulation. However, none of the analysed scenarios achieves maximum throughput, though some scenarios achieved values close to the maximum ones. Reasons for this fact are different from selected scenarios. However, the most important reasons for the failure to achieve maximum values are caused by the factors which were not analysed or those that cannot be directly influenced, those being: overhead, environment through which the signal travels, the technology used, sharing signal, interference, cell distance, mobility, transmission power, path loss, free space loss, etc. The selection of LTE-Advanced channel scenario has a significant influence on performance because the selected scenario determines the manner in which modelling of the propagation characteristics of the channel between the base station and user device is conducted. The assessed model achieves good results and failure to attain maximum value depends mostly on interference as the mechanism for coordination and interference (both inter-cell and intra-cell) influence removal weren't analysed, which influence performance considerably. Also, control information has an important role in performance decrease.

\section{CONCLUSIONS}

Enhanced MIMO offers a considerable improvement of system performance which in essence depends on multiple factors. The analysed $8 \times 8$ MIMO model, implemented with SystemVue, provides early researchers access to test solutions to verify and troubleshoot early designs and monitor system performance LTE-A networks which is facilitated by replacing analog, digital and math environments with dedicated platform for electronic system level design and signal processing realization. The paper analysed influence of a considerably larger number of parameters, namely: antenna configuration, number of HARQ retransmissions, LTE-A environment scenario and the type of duplex. By modifying each of the mentioned parameter a separate test scenario was created to analyse what parameter value influences throughput and spectral efficiency the most. Apart from the individual influence of each parameter on performance the article sums up the results and based on them, in the paper have presented the parameters that have the greatest influence on the performance and the ways in which it is possible to affect them so as to achieve better results. A comprehensive analysis of results of throughput fraction and spectral efficiency with application of $8 \times 8$ MIMO scheme in LTEAdvanced network concludes that out of all factors taken into account the greatest influence on the system performance have: the selection of LTE-Advanced channel scenario with the application of relay nodes, duplex scheme selection, and SNR as all scenarios analyse throughput fraction in relation to SNR. Application of TDD duplex scheme considerably weaker network performance is achieved since simultaneous customer servicing is not enabled.

For further research it will be wise to analyse LTEAdvanced network performance using both challenges, carier aggregation and $8 \times 8$ MIMO scheme.

\section{REFERENCES}

[1] D. Astely, E. Dahlman, A. Furuskar, Y. Jading, M. Lindstrom, S. Parkvall, "LTE: the evolution of mobile broadband", IEEE Communications Magazine, vol. 4, pp. 44-51, 2009. [Online]. Available: http://dx.doi.org/10.1109/MCOM.2009.4907406

[2] 3G Americas, The mobile broadband evolution: 3GPP Release 8 and Beyond HSPA+, SAE/LTE and LTE-Advanced, Feb. 2009.

[3] "Requirements for Further Advancements for Evolved Universal Terrestrial Radio Access (E-UTRA) (LTE-Advanced)", 3GPP TR 36.913 V.9.0.0, Dec. 2009.

[4] M. F. L. Abdullah, A. Z. Yonis, "Performance of LTE Release 8 and Release 10 in wireless communications", in Proc. Cyber Security, Cyber Warfare and Digital Forensic (CyberSec) Int. Conf., 2012, pp 236-241. [Online]. Available: http://dx.doi.org/10.1109/cybersec. 2012.6246127

[5] J.Goncalves, "Simulation-Base Performance Evaluation in LTE and LTE-Advanced". [Online]. Available: https://fenix.tecnico.ulisboa.pt/.

[6] A. Z. Yonis, M. F. L. Abdullah, "Peak-throughput of LTE-Release 10 for up/down link physical layer", Int. Journal of Information \& Network Security (IJINS), vol. 1, no. 2, pp. 88-96, 2012. [Online]. Available: http://dx.doi.org/10.11591/ijins.v1i2.452

[7] Y. Gao, X. Zhang, Y. Jiang, J. Cho, "System spectral efficiency and stability of 3G networks: A comparative study", in Conf. Rec. 2009 IEEE Int. Conf. Communications, 2009, pp. 1-6.. [Online]. Available: http://dx.doi.org/10.1109/icc.2009.5199507

[8] R. Bendlin, R. Chen, A. Ekpenyong, Z. Lin, E. Onggosanusi, "Embracing LTE-A with KeyStone SoCs", White Paper, Texas Instruments, 2012.

[9] C. Lim, T. Yoo, B. Clerckx, B. Lee, B. Shim, "Recent trends of multiuser MIMO in LTE-Advanced", IEEE Communications Magazine, vol. 51, no. 3, pp. 127-135, 2013. [Online]. Available: http://dx.doi.org/10.1109/MCOM.2013.6476877

[10] Blogspot, LTE protocols - Transmission Mode 9. [Online]. Available: http://ltea-protocols.blogspot.com/2013/06/transmission-mode-9.html

[11] Q. Li, G. Li, W. Lee, M. Lee, D. Mazzarese, B. Clerckx, Z. Li "MIMO techniques in WiMAX and LTE: A feature overview", IEEE Communications Magazine, vol. 48, no. 5, pp. 86-92, 2010. [Online]. Available: http://dx.doi.org/10.1109/MCOM.2010.5458368

[12] "LTE-Advanced: The Advanced LTE toolbox for more efficient delivery of better user experience", Technical White Paper, Nokia Siemens Networks, 2011.

[13] A. Osseiran, J. F. Monserrat, W. Mohr, Mobile and Wireless Communications for IMT-Advanced and Beyond. Ed. Wiley \& Sons, 2011, pp. 285-288. [Online]. Available: http://dx.doi.org/10.1002/ 9781119976431.app5

[14] P. K. Rekhi, M. Luthra, S. Malik, R. Atri, "Throughput calculation for LTE TDD and FDD systems", White Paper, 2012.

[15] E. Dahlman, S. Parkvall, J. Skold, 4G LTE/LTE-Advanced for Mobile Broadband. Elsevier: Oxford, 2011, pp. 129-130.

[16] Z. S. Velickovic, M. Jevtovic, V. Pavlovic, "Cross-layer throughput optimization in slow fading wireless channel", Elektronika ir Elektrotechnika, vol. 19, no. 6, pp. 131-137, 2013. [Online]. Available: http://dx.doi.org/10.5755/j01.eee.19.6.2302

[17] D. McClearnon, W. Huan, "Using SystemVue to overcome 4G challenges", Agilent Technologies [Online]. Available: http://www.eetimes.com/document.asp?doc_id=1279263

[18] J. A. Aldhaibani, A. Yahya, R. B. Ahmad, "Optimizing power and mitigating interference in LTE-A cellular networks through optimum relay location", Elektronika ir Elektrotechnika, vol. 20, no. 7, pp. 7379, 2014. [Online]. Available: http://dx.doi.org/10.5755/j01. eee.20.7.3379

[19] Y. Chen, B. Walke, "Analysis of cell spectral efficiency in 3GPP LTE systems", in Proc. IEEE 24th Annual Int. Symposium on Personal Indoor, and Mobile Radio Communications (PIMRC), London, 2013, pp. 1804-1809. 